\title{
Role of aminoacidic residues inside active sites of metalloproteins
}

\author{
Ivano Bertini*, Claudio Luchinat and Luigi Messori* \\ (*) Department of Chemistry, University of Florence, Florence, Italy \\ (i) Institute of Agricultural Chemistry, University of Bologna, Bologna, Italy
}

\begin{abstract}
The role of the residues inside the active cavity of metalloproteins can be investigated by using the metal ion itself as a spectroscopic probe. For example in carbonic anhydrase the cobalt(II) substituted enzyme has allowed us to understand the role of a free histidine hanging in the cavity in lowering the $\mathrm{pK}_{\text {, }}$ of the coordinated water. Substitution of zinc with cobalt(I) in superoxide dismutase has allowed us to check the spatial arrangement of metal ligands and the mode of inhibitor binding when important residues like Arg 143 in the human enzyme are substituted through site directed mutagenesis. The cobalt(II) substituted derivative of carboxypeptidase $A$ has been used to show that molecules with carboxylate groups bind at Arg 145. Afterwards ternary complexes with metal ligands like azide can be obtained. It is proposed that the binding of a carboxylate function to the guanidinium group of Arg 145 is the key step in modulating the opening of the cavity and the access to the metal of substrates and inhibitors.
\end{abstract}

\section{INTRODUCTION}

Recently the interest of inorganic biochemists has moved to consider the role played by those aminoacidic residues not directly bound to the metal center in metalloproteins but located in its immediate surroundings. Indeed, the joint application of physico-chemical techniques for the structural determination of macromolecules as well as the possibility of preparing proteins selectively modified in specific aminoacidic residues via both chemical modification and gene engineering have permitted, in many instances, extensive inspection into the functional cavity of metalloproteins. The main goal of this type of approach is the determination of the specific contribution of each residue to the sequence of catalytic or, more in general, functional events performed by the protein.

A single aminoacidic residue may contribute to the general process of catalysis, individually or in a concerted way, through one or more of the following mechanisms:

i) substrate recognition and orientation

ii) control of substrate and inhibitor access to the active site

iii) modification of solvent properties

iv) stabilization of intermediate states

v I stabilization of transition states

vi) allosteric control

The above functions can be played taking advantage of the specific chemical properties of the $R$ side chain of the aminoacid i.e. electrical charge, acid/base properties, hydrophobicity, hydrogen bonding properties, stacking effects, etc. as well as of the general chemical properties of the whole cavity (polarity, spatial distribution of the charges, hydrophobicity, etc.).

In this article we will survey some significant examples concerning the enzymes superoxide dismutase (SOD), carbonic anhydrase (CA) and, to a larger extent, carboxypepidase $A$ (CPA). In the latter case a large number of $X$-ray diffraction and spectroscopic studies as well as extensive chemical modification and site directed mutagenesis experiments have provided a really impressive amount of information on the role played by uncoordinated residues in the overall mechanism.

\section{SUPEROXIDE DISMUTASE AND CARBONIC ANHYDRASE}

The catalytic center of the enzyme superoxide dismutase is constituted by a copper(II) and a zinc(II) Ion, about 6 A far apart, connected through an imidazolate bridge (His 61 )(ref. 1). The general structure of the site is reported in Fig. 1. 
This binuclear center is buried inside the protein and is solvent accessible only at the catalytically active copper site, through a deep channel. In cross section this channel funnels downward stepwise from a large shallow depression, about $24 \AA$ across to a deeper well about $10 \AA$ wide and $5 \AA$ deep, then narrows to less than $4 \AA$, forming a pocket just above the copper ion. Hence it follows that the catalytic activity of the enzyme is strictly correlated to the structure of this channel as far as substrate access, recognition and orientation are concerned.

In particular, Arg 143 in the human enzyme (Arg 141 in the bovine enzyme), which is located on the surface of the channel, about 7 A far apart from the copper ion, is believed to be very important in governing the enzymatic activity. Its role has been focused through site directed mutagenesis experiments; indeed, replacement of the above residue with Lys or Ile was shown not to cause any major change in the spatial structure of the chromophore (ref. 2) but to affect markedly the enzymatic efficiency of soD (ref. $3) ;$ in the two cases activity dropped respectively to $43 \%$ and $11 \%$ of that of the native system. A parallel behavior has been observed in the pattern of the affinity constants of these mutants for inhibitors like azide (the affinity constants are respectively 154 $M^{-1}$ in the wild type derivative, $63 M^{-1}$ in SOD-Lys 143 and $16 M^{-1}$ in SOD-Ile 143 ) (ref. 2 ).

Another nice example concerning the importance of uncoordinated residues in enzymatic catalysis is provided by the isoenzymes of carbonic anhydrase of type I and II. The active site of this enzyme consists of a zinc(II) ion surrounded by three His residues (His 94, His 96 and His 119)(ref. 4) and one or two water molecules (ref. 5)(Fig. 2)

The acid base properties of the chromophore have been shown to be very relevant for activity since only the alkaline form of the enzyme (tetracoordinated zinc with a coordinated hydroxide group instead of the water molecule(s)) is the effective catalytic species. Recent detailed studies on the $\mathrm{pH}$ dependent properties of the enzyme have shown that in order to account for the dependence on $\mathrm{pH}$ of the activity (ref. 6) and of the electronic spectra of the cobalt(II) substituted enzymes (ref. 7) the simultaneous contribution of two acidic groups has to be taken into account; these two groups should correspond to a zinc coordinated water molecule and to the imidazole ring of an uncoordinated His hanging in the cavity, located in the immediate surrounding of the zinc center. The exceptionally low $\mathrm{pk}_{\text {of }}$ of the coordinated water in $\mathrm{CA}$ can be accounted for only on the above basis. These findings highlight the essential role performed by non-coordinated residues in enzymatic catalysis.

\section{CASE OF CARBOXYPEPTIDASE}

Carboxypeptidase $A$, is a zinc containing enzyme, capable of acting both as a terminal peptidase or as an esterase (ref. 8). Extensive X-ray diffraction studies on the native enzyme and several metallosubstituted derivatives as well as on many adducts of the enzyme with inhibitors and pseudosubstrates have permitted deep inspection into the structure of the active site and have allowed to shed light on the role performed by the single aminoacidic residues in the the overall catalytic process (refs. 9, 10 ).

A schematic drawing of the active site of carboxypeptidase is shown in Fig. 3 . This site consists of a pentacoordinated zinc(II) ion liganded by His 69, His 196, Glu 72 (bidentate) and a water molecule. Just near this metal center lie some aminoacidic residues which have been believed essential for the general catalytic mechanism i.e. the carboxylate group of Glu 270 (ref. 11), the phenolic group of Tyr 248 (ref. 12) and the guanidinium group of Arg 145. Even other groups situated in the cavity (1.e. Arg 127, Tyr 198, Arg 71...) might participate to the general catalytic process. The architecture of the site is completed by two hydrophobic pockets which are located in the immediate surroundings of the active site and can accomodate the phenyl ring of aromatic aminoacids (namely the $S_{1}$ ' hydrophobic pocket and a secondary pocket located between the $s_{1}$ and $s_{2}$ subsites).

\section{PROPOSED ROLES FOR RESIDUES IN CATALYTIC MECHANISM}

The general mechanism for the hydrolysis of the peptide bond by CPA is thought to require the presence of three crucial groups, namely the metal and two other groups (a base $B$ and a proton donor $\mathrm{AH}$ ) whose identity has still to be conclusively ascertained.

The mechanism could consist of the following steps:

the peptide binds into the cavity of CPA in such a way that its free carboxylate group is hydrogen bonded to the guanidinium group of Arg 145 and its aromatic group is accomodated into the $S_{1}$ ' hydrophobic pocket as it appears from X-ray studies (ref.9); the carbonyl group of the peptide bond coordinates to the zinc ion possibly replacing 


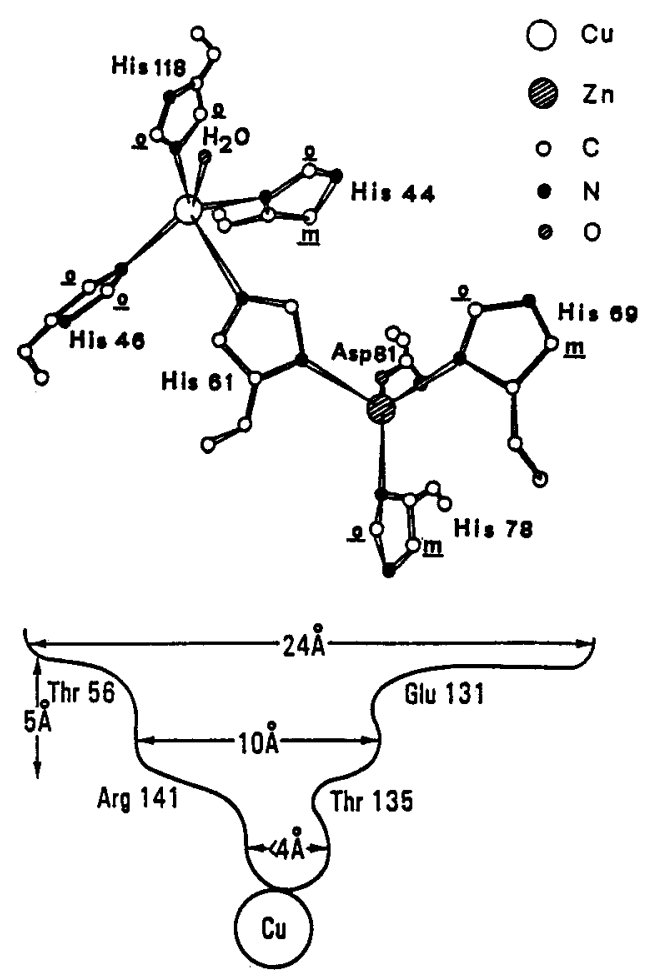

Fig. 1 The active site of bovine superoxide dismutase and a schematic drawing of the channel.

Fig. 3 probable binding mode of polypeptidic substrates to the active site of carboxypeptidase $A$.

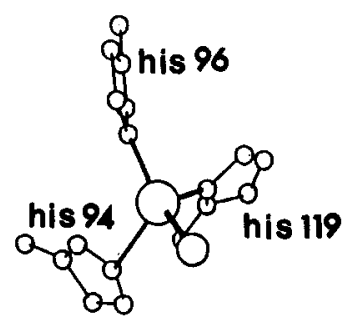

Fig. 2

The active site of carbonic anhydrase.

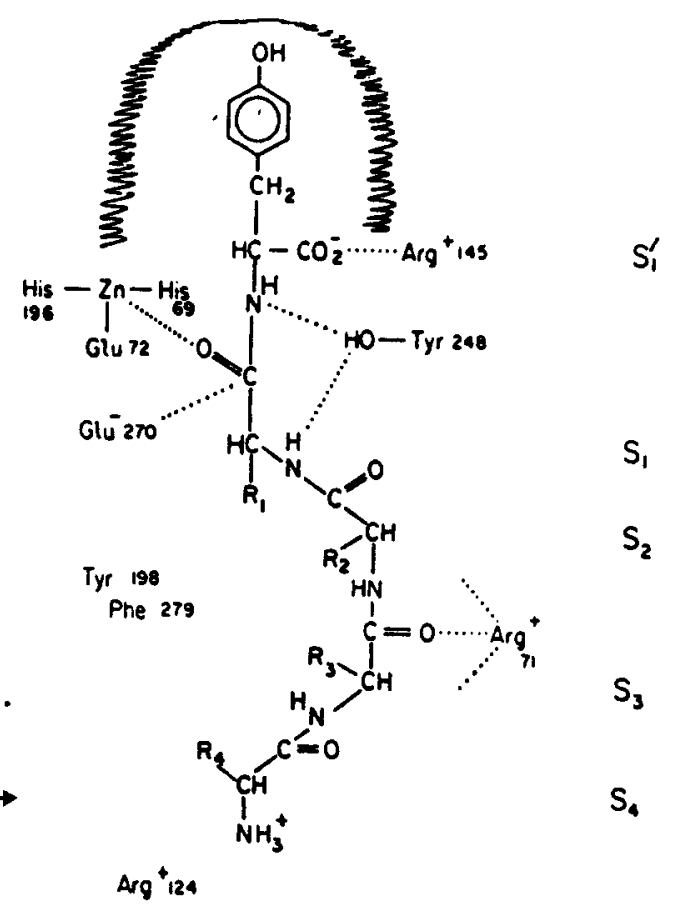

the water molecule. Zinc would act as a Lewis acid on the carbonyl group of the peptide bond, facilitating nucleophylic attack on the carbon nucleus, possibly by the carboxylate group of GIu 270 . The resulting tetrahedral transition state would then evolve towards the formation of an anhydride intermediate, which would be subsequently cleaved down by a further nucleophylic attack by a solvent molecule to give the final products.

On the basis of this proposal a number of studies have been undertaken in order to state the essentiality of the single groups, in particular the metal, 6lu 270 and Tyr 248. From these studies, mainly based on biochemical techniques of sample manipulation, it appears that both the metal and Glu 270 are indispensable for catalysis; indeed both the apoprotein (ref. 8) and a CPA derivative chemically modified on Glu 270 by carbodiimmide (ref. 13) are completely devoid of catalytic activity. More intriguing is the case of Tyr 248; for a long time this residue had been believed essential for the catalytic mechanism with the function of acting as a proton donating group; however, more recently, a CPA derivative in which Tyr 248 had been replaced by a Phe moiety through site directed mutagenesis has been shown to display a rather well conserved catalytic activity (ref. 14) so contrasting the general opinion about essentiality of Tyr 248. It should be observed anyway that the new derivative shows different $K_{M}$ values for various substrates with respect to the native enzyme, indicating some involvement of the oH group of Tyr 248 at least in the recognition of peptide substrates.

Anyway, in general, caution has to be used in dealing with such an approach; indeed, metal substitution, site directed mutagenesis and chemical modification often result in derivatives with reduced but not abolished catalytic action, different $K_{M}$, different substrate specificity, different ratio in the peptidase to esterase activities. We believe that a full understanding of the properties of the enzyme, i.e. substrate specificity, recognition and orientation, inhibition mechanism, internal dynamics of productive and non productive adducts, etc. can only arise from a direct visualization of the structure of the enzyme complexes with substrates, pseudosubstrates and inhibitors; the latter can be afforded through extensive spectroscopic and $x$-ray diffraction studies. Really, such studies may give the definitive answers to the still many controversial aspects of the chemistry of this intriguing enzyme. 


\section{CLOSER INSIGHT INTO THE CAVITY THROUGH SPECTROSCOPIC STUDIES}

The main problem in the analysis of the features of CPA and of zinc enzymes in general consists in the introduction of appropriate probes inside the cavity of the spectroscopically silent zinc enzyme, without producing any mayor structural or functional alteration.

For instance, Vallee and coworkers were able to monitor the native CPA enzyme, through absorption and CD spectroscopy, by introducing an arsanilazo probe on Tyx 248 (ref. 15). This technique allowed them to collect a lot of information on the way the enzyme interacts with substrates, hydrolysis products, inhibitors, and to map the sites at which these interactions occur. This led them to suggest the multiple overlapping site model according to which on the surface of the protein, near to the catalytic center, there are four mutually interacting sites responsible for recognition and binding of small molecules.

An even more promising way to study the fine details of the active site of CPA in solution is offered by ${ }^{1} \mathrm{H}$ NMR spectroscopy on the cobalt(II) substituted derivative (CoCPA). This spectroscopic technique permits to get direct insight into the local environment of the metal ion and to monitor the subtle changes which occur in the cavity upon interaction of the enzyme with small molecules. So, ${ }^{1} \mathrm{H}$ NMR spectroscopy, jointly to electron spectroscopy, has been fruitfully used to study the interactions in solution of the protein with carboxylate anions, aminoacids and inhibitors of pseudohalide type. These studies have allowed us to characterize and identify, out of the previously mentioned four sites, two main sites responsible for the interaction of the protein with small molecules, respectively located the first on the Arg 145 residue and the second on the metal (SITE 1 and SITE 2). Furthermore these studies permitted to clarify the stoichiometry and the structure of the adducts, and to understand their dynamic properties.

The proton MMR spectrum of CoCPA recorded at $\mathrm{pH} 6$, in $0.01 \mathrm{M}$ MES buffer, $1 \mathrm{M} \mathrm{NaCl,} \mathrm{is}$ reported in Fig. 4 A (ref. 16). It shows three isotropically shifted, quite narrow signals in the downfield region (A, B and $C$ ) which are assigned to the meta-like $\mathrm{CH}^{\prime}$ 's of His 196 and His 69 (signals $B$ and $C$ ), and to the exchangeable NH of His 69 (signal A), which is hydrogen bonded to the carboxylate group of Asp 142. The NH signal of His 196, which should lie in the same spectral region and should have similar linewidth, is not observed probably due to a higher exchange rate with bulk solvent.

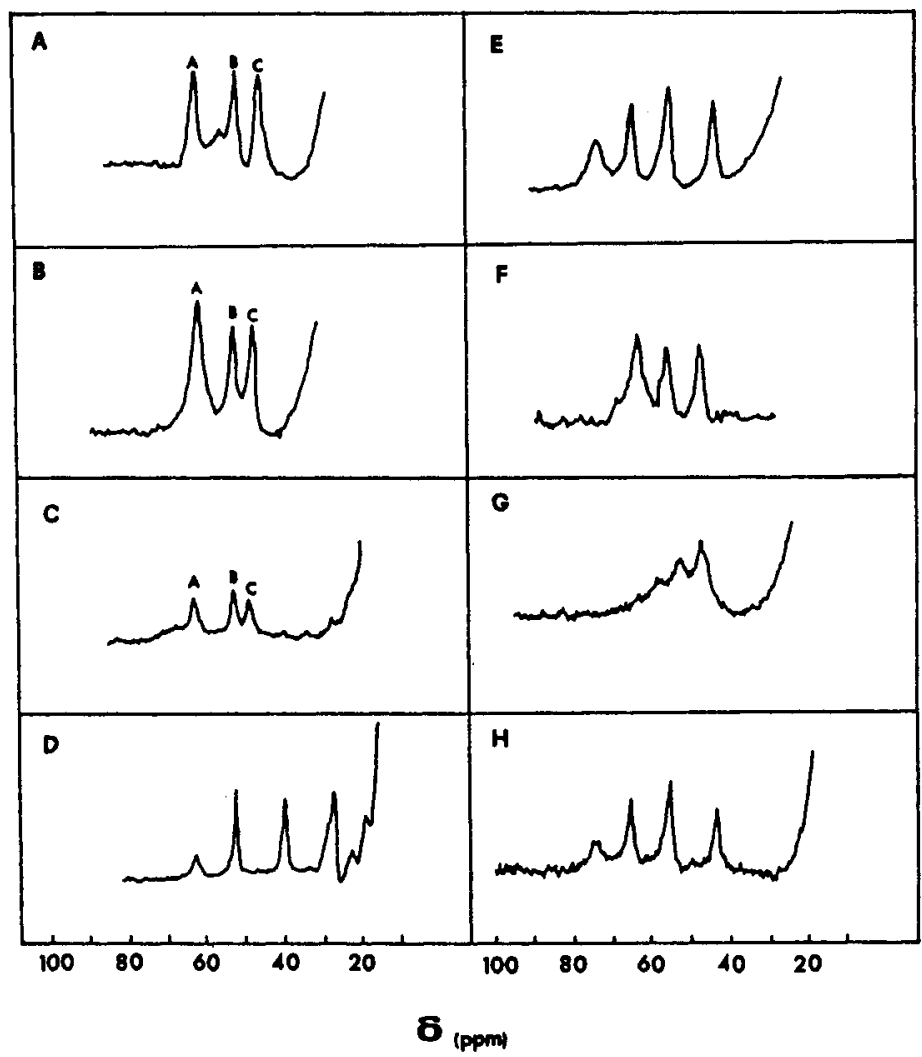

A) COCPA

B) $\operatorname{COCPA} /(\mathrm{L}-\mathrm{Phe})$

C) COCPA/(D-Phe)

D) $\operatorname{COCPA} /(\mathrm{D}-\mathrm{Phe})_{2}$

E) COCPA/L-Phe/N

F) $\operatorname{CoCPA} /$ Acetate $/ \mathrm{N}_{3}$

G) Azo-Tyz 248-CoCPA

H) Azo-Tyr 248-COCPA/L-Phe/N

Fig. 1 Proton MMR spectra in the downfield region of some CoCPA binary and ternary complexes ( $\mathrm{pH} 6,0.01 \mathrm{M}$ MES buffer, $1 \mathrm{M} \mathrm{NaCl}$ ). 
CocPA is capable of giving rise to both binary and ternary complexes with hydrolysis products and inhibitors, of general formula $\operatorname{CoCPA} / L$, $\operatorname{COCPA} / \mathrm{L}_{2}$ and $\operatorname{CoCPA} / \mathrm{L} / \mathrm{X}$, where $\underline{L}$ is a carboxylate anion or an aminoacid, and $X$ is a pseudohalide anion like $\mathrm{N}_{3}-\mathrm{SCH}^{-}$or $\mathrm{NCO}^{-}$. The stoichlometry of the binary complexes is sensitive even to the chirality of L. As an example we can refer to the case of $L$ and $D$ phenylalanine. L-phenylalanine upon interaction with CoCPA, gives rise only to a binary complex which exists under fast exchange conditions on the NMR time scale. The ${ }^{1} H$ NMR spectrum of this adduct is very similar to that of the native CoCPA system indicating that the L-Phe moiety does not bind to the metal center, but a little far apart from it, causing just slight structural changes of the metal chromophore, which remains substantially pentacoordinated (Pig. $4 \mathrm{~B}$ ). ${ }^{13} \mathrm{C}$ NMR linewidth studies on ${ }^{13} \mathrm{C}$ enriched $\mathrm{L}-\mathrm{Phe}$ (enriched on the carboxylate group) point to a distance of around 6-7 A between the carboxylate group of L-Phe and the cobalt ion; this is nicely consistent with direct binding of the free carboxylate molety to the guanidinium group of Arg 145 (SITE 1)(ref. 17). High concentrations of $\mathrm{L}-\mathrm{Phe}$ in solution do not cause any further variation of the electronic and NMR spectra. This view is supported by a low resolution X-ray report (ref. 18).

on the other hand, D-Phe shows a biphasic behavior (ref. 16). Addition of low amounts of D-Phe to a CoCPA solution results in slight proton NMR spectral changes which parallel those observed for $L-P h e$ (Fig. $4 \mathrm{C}$ ). Again we observe formation of a $1: 1$ adduct under fast exchange conditions with an affinity constant just a little higher than in the previous case $\left(690 \mathrm{M}^{-1}\right.$ versus $\left.290 \mathrm{M}^{-1}\right)$; the binding site should be exactly the same as for L-Phe (SITE 1). By increasing D-Phe concentration we observe dramatic changes with respect to $\mathrm{L}-\mathrm{Phe}$. Indeed, for D-Phe concentration higher than .01 $\mathrm{M}$, CoCPA (1 mM) starts binding a second D-Phe moiety at its active site. This behavior can be easily followed through proton MMR spectra, which show the appearance of a new species, with quite distinct spectral features, under slow exchange conditions. The second species, whose MMR spectrum is shown in Fig. $4 \mathrm{D}$, should correspond to a CoCPA/(D-Phe) 2 species with the first D-Phe moiety bound to the $s_{1}^{\prime}$ site, and the second D-Phe moiety directly bound to the metal through its carboxylate group. The structure of this adduct should be very similar to that found through $x$-ray diffraction studies for the ternary adduct between native CPA, the amide substrate $\mathrm{N}$-benzoyl-L-phenylalanine and $L$-phenylalanine (ref. 19).

SITES 1 and 2 have been shown to be structurally and functionally correlated. This is very clear in the case of the ternary complexes with pseudohalides where strong cooperative effects in the binding of aminoacids and pseudohalides have been observed. Indeed, the presence of $L$ in the $S_{1}$ ' pocket enhances the affinity of pseudohalides for the metal by orders of magnitude. The mechanism through which this occurs could be both conformational and electrostatic. Binding of $\mathrm{L}-\mathrm{Phe}$, for instance, to Arg 145 could result in an opening of the metal site mediated through a conformational change of the peptide backbone as well as in the screening of the negative charge located on Glu 270 (formation of a salt linkage with the positive ammonium group of the aminoacid) and subsequent disruption of the hydrogen bond interaction of Glu 270 with the metal coordinated water; this would facilitate the entrance of a negatively charged $x$ group.

The ternary complexes CoCPA/L/X, with $L=D$ or L-Phe, exhibit very similar spectral properties independently on the nature of $X$ (see Fig. 4 E)(ref. 16); in these adducts the cobalt(II) chromophore appears to be pseudotetrahedral, the donor atoms being two nitrogens from His 69 and $\mathrm{His} 196$, one oxygen from the carboxylate group of $\mathrm{Glu} 72$ and the $x$ moiety. It is possible that upon formation of the ternary complex $z$ inc moves from the original position causing the detatchment of an oxygen of Glu $72 .{ }^{35} \mathrm{Cl}$ NMR studies have shown that chloride interacts with the protein, possibly with the metal, in presence of L-Phe. Azide removes chloride from this interaction (ref. 20). This finding is meaningful because CPA is soluble only at high ionic strenght generally provided by chloride salts at concentrations larger than $1 \mathrm{M}$.

Further evidence comes from parallel proton NMR studies performed on the arsanilazo Tyr 248/CoCPA system (Azo-Tyr 248-CoCPA). Indeed, chemical modification of CoCPA at the Tyr 248 residue results in a clear spectral change with respect to the native CoCPA system (Fig. (G). In particular we observe the disappearance of signal $A$, whereas signals $B$ and $C$, which are almost at the same place as in the native system, exhibit much broader linewidths, indicating an increased fluxionality of the chromophore. Anyway, addition of L-Phe and azide to this Azo-Tyr 248-CoCPA derivative results in the formation of a derivative with proton NMR spectral properties absolutely identical to those of the COCPA/L-Phe/N $\mathbb{N}_{3}$ adduct (Pig. $1 \mathrm{H}$ ). This means that the ternary complex is formed again with the same structure as before and that the azo group is displaced from coordination to the cobalt(II) ion (ref. 20 ).

Similar ternary complexes of CoCPA with azide can be prepared even if $L$ is a carboxylate anion like acetate, $\beta$-phenylpropionate or phenylacetate (see the proton NMR spectrum of the adduct CoCPA/acetate $/ \mathbb{N}_{3}$ in $\mathrm{Fig} .4 \mathrm{~F}$ ). The structure of such complexes appears to be almost identical from one another and substantially resemblant of that of the ternary complexes with aminoacids in the sense that $L$ again is located in SITE 1 and the $X$ anion is coordinated to the metal. Again the binding of both $L$ and $X$ exhibits a clear positive 
cooperativity. Structural differences with respect to the ternary complexes with aminoacids could arise from the circumstance that in the latter case no positive ammonium group is avallable to screen the negative charge of the $\mathrm{COO}^{-}$group of Glu 270 .

\section{RELEVANCE OF THE TWO-SITE MODEL TO CATALYTIC MECHANISM}

The above reported two-site model, even if necessarily schematic, may be very useful in rationalizing the general modes of interaction of the protein with substrates, pseudosubstrates, inhibitors. Furthermore it is particularly proficuous in illuminating the structural flexibility of the protein in solution. Indeed, proteins, in constrast to the blocked picture offered for a long time by $x$-ray crystallography, have to be considered as extremely dynamic structures with high internal mobility. Interaction with small molecules causes important local conformational changes which bring the whole system to a general spatial arrangement favorable for the catalytic process to occur, as stated by the induced fit model. This is very clear in our case. Indeed, SITES 1 and 2 appear to be strictly correlated each other in the sense that the formation of a salt linkage at the guanidinium group of Arg 145 (SITE 1) upon interaction with the carboxylate function of the ligand triggers a conformational change at SITE 2 (i.e. the metal site) consisting in the transition from a closed inactive form to an open, substrate accessible and catalytically active form; so the electrostatic interaction which takes place at SITE 1 appears really to be the starting signal for the catalytic event.

\section{CONCLUDING REMARKS}

It appears clear now that although the metal can be the catalytic center in metalloproteins in the sense that without it the protein does not show any residual catalytic activity, other groups inside the cavity play important and specific roles. Positive residues (His in most CA's and Arg in the novel CA of type III) account for the low $\mathrm{pk}_{\mathrm{a}}$ of the coordinated water and assist the proton transfer process in the step from coordinated water to coordinated hydroxide (ref. 5). Arg 143 in human soD attracts anions and possibly superoxide inside the cavity. Its replacement with a neutral residue like Ile reduces the activity to about one tenth of the original value; replacement with a negative residue like Glu eliminates it completely (ref. 20). In CPA the metal ion becomes accessible to anions only after a carboxylate group binds Arg 145 . We are proposing that the binding of substrates to Arg 145 is the first step which allows the carbonyl group to bind the metal.

\section{REFERENCES}

1. J.A. Tainer, E.D. Getzoff, K.M. Beem, J.S. Richardson and D.C. Richardson, J. Mol.Biol., 160, 181-217 (1982).

2. L. Banci, I. Bertini, C. Luchinat and R.A. Hallewell, submitted.

3. R.A. Hallewell, R. Mills, P. Tekamp-olson, R. Blacher, S. Rosenberg, F. Otting, F.R. Masiarz and C.F. Scandello, Biotechnology, in press.

4. K.K. Kannan, B. Notstrand, K. Fridborg, S. Lovgren, A. Ohlson and M. Petef, Proc. Natl. Acad. Sci. U.S.A., 72, 51-55 (1975).

5. I. Bertini and C. Luchinat, ACC. Chem. Res., 16, 272-279 (1983).

6. I. Simonsson and S. Lindskog, Eur. J. Biochem., 123, 29-36 (1982).

7. I. Bertini, A. Dei, C. Luchinat and R. Monnanni, Inorg. Chem., 24, 301-303 (1985).

8. B.L. Vallee, A. Galdes, D.S. Auld and J.F. Riordan, in "zinc Enzymes", Wiley New York, 25-75 (1983).

9. D.W. Christianson and W.N. Lipscomb, in "zinc Enzymes", Birkhauser Boston, $\underline{8}$, 121-132 (1986).

10. D.C. Rees, J.B. Howard, P. Chakrabarti, T. Yeates, B.T. Hsu, K.D. Hardman and W.N. Lipscomb, in "Zinc Enzymes", Birkhauser Boston, 10, 155-166 (1986).

11. M.E. Sander and H. Witzel, in "Zinc Enzymes", Birkhauser Boston, 13, 207-214 (1986).

12. D.S. Auld, K. Larson and B.L. Vallee, in "zinc Enzymes", Birkhauser Boston, g, 133-154 (1986).

13. K.F. Geoghegan, B. Holmquist, C.A. Spilburg and B.L. Vallee, Biochemistry, 22, 1847-1852 (1983).

14. S.J. Gardell, C.S. Craik, D. Hilvert, M.S. Urdea and W.J. Rutter, in "Zinc Enzymes", Birkhauser Boston, 12, 191-206 (1986).

15. G.M. AIter and B.L. Vallee, Blochemistry, 17, 2212-2218 (1978).

16. I. Bertini, B.L. Vallee, et al., in preparation.

17. C. Luchinat, B.L. Vallee, et al., in preparation.

18. T.A. Steitz, M.L. Ludwig, F.A. Quiocho and W.N. Lipscomb, J. Biol. Chem., 242, 4662-4668 (1967).

19. D.W. Christianson and W.N. Lipscomb, grivate communication.

20. Unpublished results from this laboratory. 